\title{
OXYGEN CONSUMPTION AND MOTILITY OF MOUSE SPERM AS AFFECTED BY OXIDIZABLE SUBSTRATES AND OXYGEN TENSION
}

\author{
by \\ E.J. BOELL and J.K. BURKUS \\ Department of Biology, Yale University, \\ P.O. Box 6666, Yale University, New Haven, Ct. 06511
}

Keywords: Sperm, oxygen consumption, motility, energy sources, oxygen tension

The rates of oxygen consumption and motility of washed sperm from the cauda epididymidis of the mouse declines steadily during the course of three or more hours. The decrease can be prevented by including an oxidizable substrate in the medium. Of the substrates usually included in media in which sperm are incubated, pyruvate and lactate were equally effective in maintaining respiratory rate and motility at maximal levels; glucose or fructose increased respiration only slightly, although motility was maintained by the hexose sugars as well as when pyruvate or lactate were present. Presumably then, the production of ATP by aerobic glycolysis provides the energy necessary for motility.

Both the rate of oxygen consumption and the level of motility are positively correlated with the partial pressure of oxygen in the medium. Motility, at a level about $20 \%$ of maximum, persists when oxygen consumption ceases due to oxygen lack. This occurs even though the medium contains no glucose. The most plausible explanation of this is that endogenous sources contribute to the production of energy.

\section{INTRODUCTION}

The results of numerous investigations, by workers throughout the world, lead to the conclusion that mammalian spermatozoa derive their energy primarily through aerobic metabolic activity. However, under conditions in which oxygen is lacking or is present in amounts insufficient to support respiration, sperm are also capable of facultative anaerobiosis, so that they then obtain energy through glucolysis or, especially, fructolysis (19).

Suggestions may be found in the current literature that human sperm synthesize ATP through glycolysis, even under conditions in which oxygen is present and respiration is occurring $(6,21,25)$. Mouse sperm have been reported to require glucose (15) or a glycolytic product (10), for which lactate and pyruvate will not substitute, in order to complete capacitation and the acrosome reaction. The motility of hamster sperm seems to be unaffected by the presence or absence of glucose, provided that other substrates are present, but activation and the acrosome reaction are improved when glucose is added to the medium (7).

By contrast, glucose has been reported to inhibit the acrosome reaction of guinea pig sperm (23). Other studies have shown that the 
sperm of man $(9,24)$, mouse, rabbit, and bull (5) carry on vigorous oxidative phosphorylation to produce the ATP needed for their energetic requirements, and that oligomycin, antimycin$A$, and rotenone - agents that inhibit oxidative phosphorylation - reduce the ATP content of human (24) and of hamster sperm (22).

Most of what is known about the metabolic activities of sperm has been derived from studies conducted in vitro - an environment in which sperm are normally never found - and there may be question, therefore, as to whether sperm behave metabolically in vivo as they do in vitro.

A priori considerations, however, as well as indirect experimental evidence, support the notion that the energetic needs of mammalian sperm are normally met through oxidative phosphorylation. Among them may be mentioned the meticulous conservation of spermatid mitochondria and their sequestration in the middle piece of the sperm tail during the terminal phases of spermiogenesis, processes that run counter to the general tendency during spermiogenesis for all excess cytoplasm and useless cytoplasmic organelles to be sloughed off in order to reduce the bulk of the sperm as much as possible. In addition, the location of the mitochondrial apparatus in the middle piece of the sperm tail insures efficient transfer of ATP - the product of oxidative phosphorylation - to the contractile elements through which motility is achieved in the sperm flagellum (17). FAWCETT has pointed out, in this connection, that a correlation may exist between the number, size, and arrangement of mitochondria and the energetic needs of the spermatozoa possessing them (8). Fianally, may be mentioned the fact that the partial pressure of oxygen in the female reproductive tract, in which the sperm fulfill their intended function, is not appreciably different from that of other active tissues of the body $(12,26)$. Sperm at this partial pressure of oxygen can carry on active oxidative processes, as they are transported from the point of entry in the female reproductive tract to the site of fertilization.

The experimental work in the present project, stimulated by consideration of some of the questions or implications arising from the published reports cited above, were designed in particular to examine the relationship between sperm metabolism and motility - the primary energy-requiring activity - as influenced by such factors as age of the sperm, the presence or absence of substrates and their nature, and by variations in oxygen tension.

\section{MATERIALS AND METHODS}

Sperm were obtained from the caudae epididymides (CE) and the vasa deferentia (VD) of 3- to 4-month old mice (Mus musculus) of various genetic strains. No attempt was made to segregate the data according to strain, for variation within strains was as great as that among them.

Mice were killed by cervical dislocation; the CE's and VD's were dissected free and placed in a watch glass containing $0.9 \% \mathrm{NaCl}$ solution. Fat, connective tissue, and blood vessels were removed with watchmaker forceps, and the cleaned organs were then transferred through two washes in saline solution to $0.5 \mathrm{ml}$ of the medium in which sperm were to be isolated.

Sperm were stripped from the VD's by passing them through the tips of barely closed watchmaker forceps. Two longitudinal and four transverse cuts were made in each $\mathrm{CE}$, with iridectomy scissors, and sperm were then eased out of the epididymal tubules by pressure from forceps or a fine stainless steel spatula. The watch glass was then placed in a covered plastic box, equipped with entrance and exit ports, so that air or other desired gas mixture could be run through in order to equilibrate the medium. Before entering the gassing chamber, the gas was bubbled through a wash bottle, containing medium, in order to saturate it with water vapor and thus to prevent evaporation from the sperm suspension. The chamber was then placed in an incubator at $37^{\circ} \mathrm{C}$ for 15 to $20 \mathrm{~min}$ in order to permit sperm to migrate from the cut ends of the epididymal tubules.

After this, the sperm suspension was sucked into a fine pipette, care being taken to avoid tissue fragments, and transferred to a small test tube. The tissue in the watch glass was rinsed with $0.5 \mathrm{ml}$ of medium and the washings added to the tube. The suspension was centrifuged at $55 \times \mathrm{G}$ for $5 \mathrm{~min}$, to sediment tissue fragments and some immotile sperm, and the clean suspension was then withdrawn for use. 
In some cases, sperm were washed free of epididymal juices by centrifuging at $475 \times \mathrm{G}$ for $5 \mathrm{~min}$. The medium was removed by aspiration, replaced by fresh medium, and the pelleted sperm then resuspended by passing them in and out of a fine pipette. Centrifugation was repeated one or more times and fresh medium added after each. Prepared in this way, the suspensions generally contained approximately $7 \times 10^{6}$ sperm $/ \mathrm{ml}$.

Various media were used, most being similar in composition to Tyrode's solution to which various substrates could be added as desired, and the bicarbonate concentration varied, depending upon the gas mixture used and the method of measuring respiration. The usual concentrations of substrates were $5.56 \mathrm{mM}$-glucose or fructose, $25 \mathrm{~mm}$-lactate, and 0.5 to 10 mM-pyruvate. Bovine serum albumin was added to most preparations in a concentration of $3 \mathrm{mg} / \mathrm{ml}$; in some cases, $1 \mathrm{mg}$ or $12 \mathrm{mg} / \mathrm{ml}$ were used.

Oxygen consumption of the sperm suspension was determined by means of Cartesian diver technique $(1,18)$ (see footnote*) and a polarigraphic method, in which a Clark type oxygen electrode was used in a specially constructed chamber permitting gassing of the chamber and the introduction of reagents during the course of an experiment (2).

With the Cartesian diver technique, divers of $10-12 \mu \mathrm{l}$ capacity were used. Generally 1 or $0.5 \mu \mathrm{l}$ of sperm suspension was pipetted into the diver bulb and, seriatim, into the neck, 1 $\mu \mathrm{NaOH}, 1 \mu \mathrm{loil}$, and the neck seal. The initial experiments were done at $25^{\circ} \mathrm{C}$; for the later experiments, a temperature-controlled chamber of lucite was constructed in which the diver filling apparatus, the sperm suspension, and all reagents could be kept at $37^{\circ} \mathrm{C}$. The filled divers were then transferred to a $37{ }^{\circ} \mathrm{C}$ bath. All

* The Cartesian diver technique, in recent years, has fallen into disuse perhaps, primarily, because to take advantage of its many excellent features, skill, patience, and concern for what may appear to be trivial technical details are required. When properly used, the technique is completely reliable and is of particular utility when the oxygen uptake of small samples of material is to be followed for periods of several hours. experiments with the oxygen electrode respirometer were performed at $37^{\circ} \mathrm{C}$.

Counts of the sperm in each diver were made at the end of an experiment. Neck, oil, and alkali films were removed by suction; the diver neck wiped with a twist of toilet tissue, and medium was then pipetted into and out of the bulb until all sperm had been recovered. The volume of the medium used to wash out the diver was determined by weighing in order to determine the dilution. Sperm were then counted with hemocytometer. With the oxygen electrode apparatus, an aliquot of the sperm suspension was drawn from stock before the experiment or on a sample withdrawn from the respirometer at the end. These were appropriately diluted and the sperm counted. Respiratory rate was calculated as ${ }^{\mathrm{z}} \mathrm{O}_{2}$ ( $\mu$ l oxygen consumed $/ 10^{8}$ sperm/ hour).

In the initial experiments, motility was rated by a single three-step scale: +++ , highly active; ++ , moderately active; + , sluggish or non-motile. For later work, a more sophisticated method was devised. Each sperm in a representative sample was catalogued as motile or non-motile, and the percentage motility calculated. In addition, the quality of motility of the individual sperm was determined. These scores, when added and divided by the number of sperm examined, gave an average for the sample.

\section{RESULTS}

\subsection{Effect of metabolic substrates on respiration and motility}

The sperm in a fresh suspension from the CE, in addition to their endogenous substrates, obtain exogenous, metabolizable materials from the secretions of the CE. If the sperm are not washed, these are carried over into the suspending medium. The exogenous, epididymal substrates can maintain respiratory activity at maximal, or nearly maximal levels for a few minutes, but they are soon exhausted. Oxygen uptake at the expense of endogenous substrates can continue, however, at a low, constant rate for several hours (Curve A, Figure 1). HAMMERSTEDT (11) has estimated that endogenous substrates account for about $12 \%$ of the oxygen uptake of sperm. 


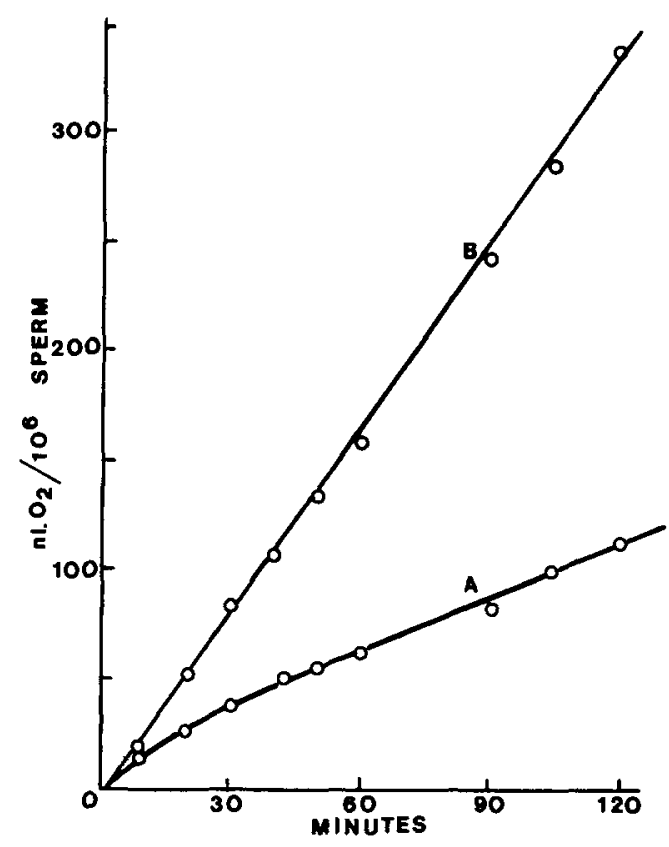

Figure 1. Respiration of sperm as measured in the Cartesian diver apparatus at $25^{\circ} \mathrm{C}$. A: Sperm in bicarbonate-free Tyrode's solution (salts only). $12 \times 10^{4}$ sperm/diver. B: Sperm washed by centrifugation and suspended in same solution as in $A+5 \mathrm{~mm}$-pyruvate $+1 \mu \mathrm{g} / \mathrm{ml}$ BSA. Ordinate $\mathrm{nl} \mathrm{O}_{2} / 10^{6}$ sperm; abscissa, time in minutes.

The decrease in the rate of oxygen consumption can be prevented by adding a substrate, such as pyruvate or lactate, to the washed sperm. When this is done, respiration then continues at a steady rate for several hours (Curve B, Figure 1).

Addition of glucose or fructose to a sperm suspension increases the rate of oxygen uptake slightly, if at all, owing to the operation of the Crabtree effect, first noted by LARDY and PHILLIPS (16) with bull sperm more than forty years ago. When this occurs, the spermatozoa break down hexose to lactic acid, via a glycolytic pathway, even when air is present, but the lactate presumably is not oxidized until the concentration reaches an optimal level (Curve A, Figure 2). When pyruvate or lactate are added simultaneously with fructose or glucose, the oxygen consumption of the sperm suspension is as rapid as it is with pyruvate or lactate alone (Curve B, Figure 2). The results of experiments with

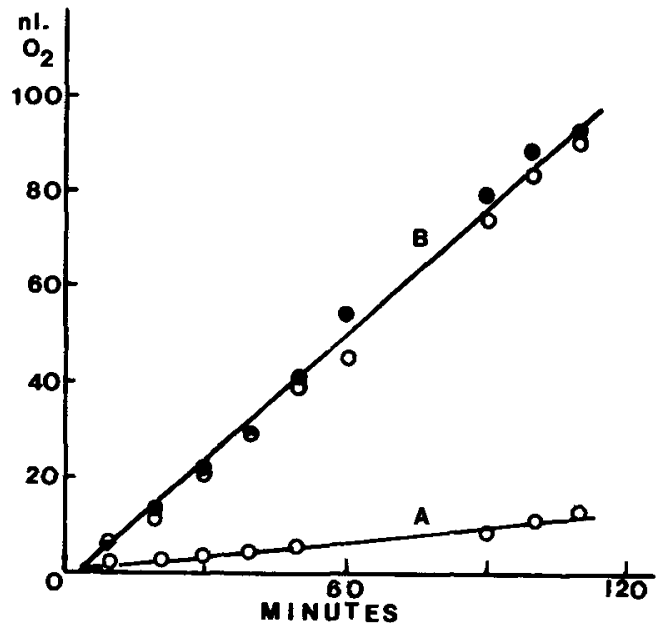

Figure 2. Respiration of washed sperm in bicarbonatefree Tyrode's solution plus added substrates as shown. Diver apparatus, $25^{\circ} \mathrm{C}$. A: Fructose. $27.4 \times 10^{4}$ sperm/ diver. B: Open circles. Fructose + pyruvate. $23.7 \times 10^{4}$ sperm/diver. B: Closed circles. Fructose + lactate. $23.7 \times 10^{4}$ sperm/diver.

the oxygen electrode respirometer show that the reduced respiration of sperm, in the presence of glucose, is abolished as soon as pyruvate or lactate is added (2). When glucose or fructose represent the only exogenous substrates available, the Crabtree effect operates; when glucose or fructose are added together with pyruvate or lactate, or in the presence of exogenous substrates from the $\mathrm{CE}$, no effect, or only minimal effect is noted.

The following experiment with the diver apparatus is illustrative. A small ribbon of sperm was extruded into the desired medium from an epididymal tubule. The sperm were quickly sucked into a calibrated, siliconed $1 \mu$ l pipette in medium and introduced into the bulb of a diver. The $C E$ was then transferred to a medium of different composition where another small ribbon of sperm was extruded and transferred to a second diver. This process was repeated until five divers, each containing sperm from the same CE, but with a different substrate, were prepared. Oxygen consumption was measured over the course of two hours, the sperm were then flushed out of the divers and counted. The result of this experiment, typical of others of the same type, is shown in Table I. There is 
Table I.

Oxygen consumption of sperm from the cauda epididymidis in the absence or presence of exogenous substrates. (Diver experiment, $37^{\circ} \mathrm{C}$ ).

\begin{tabular}{lcll}
\hline Substrate added & ${ }^{\mathrm{x}} \mathrm{O}_{2}$ & $\begin{array}{l}\text { Sperm count } \\
\times 10^{4}\end{array}$ & ${ }^{\mathrm{z}} \mathrm{O}_{2}$ \\
\hline None & 25.0 & 7.35 & 34 \\
P, L, G & 13.2 & 3.15 & 42 \\
L & 20.6 & 5.25 & 39 \\
P & 37.0 & 9.25 & 40 \\
G & 3.1 & 0.84 & 36 \\
\hline
\end{tabular}

G- 5.56 mM-d-glucose; L- 20mM-pyruvate; P- $5 \mathrm{~mm}$ pyruvate; substrates from $\mathrm{CE}$ secretions are also present in all divers. ${ }^{\mathrm{x}} \mathrm{O}_{2}=\mathrm{nl}$ oxygen consumed per hour by the number of sperm counted.

no significant difference in the oxygen uptake of any of the sperm samples, the exogenous substrate contributed by the CE being as effective as pyruvate or lactate in abolishing the Crabtree effect. Different results are obtained with washed sperm. A summary of the respiratory activity of unwashed and washed sperm in the absence of substrates and in the presence of added metabolites, is included in Table II. In the cases shown, the respiration of sperm

\section{Table II}

Summary of respiratory rates of washed sperm in the presence of various substrates. (Oxygen electrode respirometer, $37^{\circ} \mathrm{C}$ ).

\begin{tabular}{lll}
\hline $\begin{array}{l}\text { Substrate added } \\
\text { Group I }\end{array}$ & $\mathrm{N}$ & ${ }^{\mathrm{z}} \mathrm{O}_{2}$ \\
\hline None & 7 & 15 \\
Fructose & 2 & $19.2 \pm 0.9$ \\
Glucose & 4 & $17.5 \pm 2.0$ \\
Sucrose & 2 & 15.9 \\
Acetate & 1 & 14.4 \\
\hline Group II & & \\
\hline Lactate & 3 & $44.1 \pm 2.6$ \\
Pyruvate & 3 & $32.1 \pm 3.6$ \\
G, L, P & 45 & $42.0 \pm 1.5$ \\
\hline
\end{tabular}

The difference between any two values in group 1 or between any two values in group II is not statistically significant. Any value in group II is significantly different from any value in group $\mathrm{I}$. $\mathrm{N}=$ number of determinations.

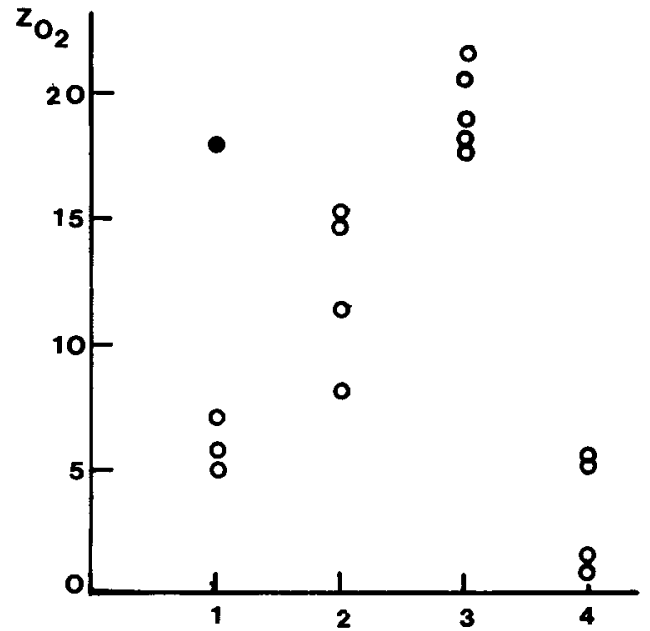

Figure 3. Respiratory rate of sperm samples varying in motility. Ordinate $-{ }^{2} \mathrm{O}_{2}\left(\mu l \mathrm{O}_{2} / 10^{8}\right.$ sperm $/ \mathrm{hr}$. Abscissa - 1 . sluggish or non-motile, 2 . moderately active, 3 . highly active, 4 . highly active sperm in the presence of fructose.

deprived of substrates by washing is low. The addition of glucose or fructose to such sperm suspensions results in minimal increase in the rate of oxygen consumption. But lactate and pyruvate, alone or in combination with glucose, produce marked increase of respiratory rate over that of washed sperm.

During the course of this investigation it was noted that sperm samples differed greatly both in the number of motile sperm and, in those sperm that were motile, in the vigor of their flagellar movements. In the initial experiments, such variations in motility were scored as highly active, moderately active, and sluggish or nonmotile. The results of these observations, in spite of the crudeness of the motility ratings, show a positive correlation between the rate of oxygen uptake and the quality of motility. Interestingly, they also show that when fructose was the only substrate present and respiratory activity was, therefore, low, the sperm in this group were as active as those in group 3 (Figure 3).

The motility of sperm declines with time, an observation reported frequently by others. Even in the presence of added substrate, the rate declines somewhat, although after two hours, it is still $90 \%$ of the initial value (Curve A, Figure 4). In these experiments and those that follow 


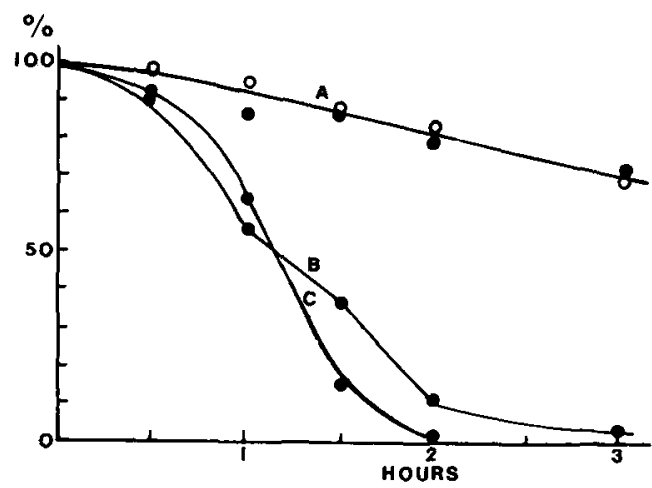

Figure 4. Temporal decline in motility and oxygen consumption of sperm. A: Open circles. Motility of unwashed sperm + pyruvate, lactate, and glucose. Closed circles: motility of washed sperm + substrates listed in A. B: Motility of washed sperm, no substrate. C: Oxygen consumption of washed sperm, no substrate. Ordinate, percentage of motility or oxygen consumption; $100 \%=$ value at time zero. Abscissa, time in hours.

motility was rated by a more quantitative method (See Materials and Methods). With a washed sperm suspension, in the absence of exogenous substrates, the decline in motility is rapid. The rate, one hour after isolating the sperm, is $50 \%$ of the initial rate; at two hours, $15 \%$, and at three hours, only 5\% (Curve B, Figure 4). If sperm are provided with an oxidizable substrate within two hours after washing, substantial recovery of motility is possible; starvation for three hours, however, leads to irreversible damage. At the end of a three-hour period, only a few sperm continue to be active, and these show only feeble movements of their flagella, so that, instead of swimming, the sperm merely quiver in place.

The respiratory rate of a washed sperm suspension shows a similar decline with time (Curve C, Figure 4), and the correlation coefficient between decrease in respiratory rate and decrease in motility is $\mathbf{0 . 9 7 8}$.

\subsection{Motility and respiration as affected by oxygen tension}

The normal milieu in which sperm are found in vivo has a partial pressure of oxygen of about $50 \mathrm{~mm} \mathrm{Hg}(12,26)$. At this oxygen tension in

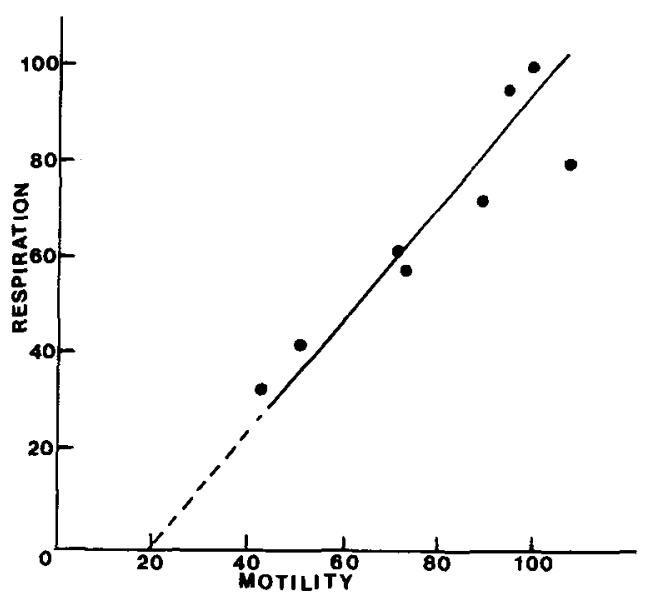

Figure 5. Correlation between decrease in respiratory rate and motility of sperm with decreasing oxygen tension, between ca., $160 \mathrm{~mm}$ and $7.5 \mathrm{~mm}$. Ordinate, percentage ${ }^{2} \mathrm{O}_{2}$ in air. Abscissa, percentage of motility value in air. Motility measured at $23{ }^{\circ} \mathrm{C}$; oxygen consumption at $25^{\circ} \mathrm{C}$. Coefficient of correlation between variables is 0.939 .

vitro, sperm show vigorous motility only slightly less than that in a medium saturated with air. But as oxygen tension is further reduced, motility also is reduced. This close correlation between motility and oxygen consumption, as both are influenced by oxygen tension, can be seen in Figure 5. The curve intercepts the abscissa at about $20 \%$ motility, indicating that, although respiration has ceased, motility at a low level still persists, probably from the energy obtained from anaerobic utilization of endogenous substrates.

\section{DISCUSSION}

The data presented in the foregoing paragraphs point clearly to the fact that sperm can carry on an active oxidative metabolism at oxygen tensions even lower than those normally encountered in vivo. By adding to the medium such substrates as lactate and pyruvate, separately or in combination with each other or with glucose, sperm can be kept in a highly motile state for six hours or longer - far more time than is needed for sperm to be transported along the reproductive tract to the site of fertilization (4). 
The experimental results reported show that the motility of mouse sperm is supported by oxidative processes, a close relationship existing between the degree of motility and the level of oxygen consumption. The persistence of motility in the presence of glucose when respiration is low or even in the absence of respiration, under conditions of greatly reduced oxygen supply, suggests that the sperm can call upon glycolytic mechanisms to supply the energy needed.

Although the data obtained provide a consistent picture of the relationship between metabolism and motility, that relationship appears to be far from a simple one. Samples of sperm are occasionally found that have low motility but in which respiration proceeds at the high rate characteristic of highly active sperm. An example is shown by the solid point in Figure 3. The occurrence of sperm samples with high respiratory rate, but low motility, is perplexing, but it probably does not represent loss of viability for immotile sperm can frequently be stimulated to motility by treating with caffeine. HARRISON et al. (13) also conclude that low motility does not necessarily indicate reduced viability.

Sometimes the motility of a given suspension, with good initial motility, almost ceases without an appreciable effect on respiratory rate resulting. MORTON et al. (20) have pointed out that, in the case of hamster sperm, the respiratory rates after the induction of motility from quiescence were not significantly different from those of the sperm before movement was initiated and that sperm with a motility rating of $30 \% / 4$ consumed as much oxygen as those with a rating of 70\%/9 Similarly, HEFFNER and STOREY (14) have stated that under certain circumstances motile mouse sperm consume no more oxygen than immotile sperm. That others may have encountered similar cases of sperm samples in which motility was less than normally expected is implicit in such statements as only sperm with high motility were used, or sperm specimens with high initial motility were selected.

Finally, may be mentioned the observation that the non-motile sperm from the caput or corpus epididymidis of the mouse respire at the same or slightly higher rate than those from the cauda; in addition, the cytochrome oxidase activity of corpus or caput sperm is higher than that of the cauda (3). It seems clear that much will yet be learned and much written on the relationship between the motility and metabolism of the mammalian sperm.

\section{ACKNOWLEDGEMENT}

This research was supported by a grant to the senior author from the Whitehall Foundation.

\section{REFERENCES}

1. Boell. E.J.: The Cartesian diver technique in microrespirometry and enzyme assay. Ch. 8 in Nematology, N.J. Sasser and W.R. Jenkins, eds. University of North Carolina Press, Chapel Hill (1960)

2. BOELL. E.J.: The oxygen consumption of mouse sperm and its relationship to capacitation. J. Exp. Zool., in press (1984)

3. BoELL. E.J.: unpublished data

4. Blandau, R.J.: Gamete transport. In: The Mammalian Oviduct: Comparative Biology and Methodology, pp. 129-162, Table 4, E.S.E. Hafez and R.J. Blandau, eds., The University of Chicago Press (1969)

5. Carey, J.F.. D. Olds-Clarke\& B.T.STorey: Oxidative metabolism of spermatozoa from inbred and random bred mice. J. Exp. Zool. 216, 285-292 (1981)

6. Chulavatnol, M. \& A. Haesungcharern: Stabilization of adenylate energy charge and its relation to human sperm motility. J. Biol. Chem. 252, 8088-8091 (1977)

7. Dravland. E. \& S. Meizel: Stimulation of hamster sperm capacitation and acrosome reaction in vitro by glucose and lactate and inhibition by the glycolytic inhibitor $\alpha$ - chlorohydrin (1981)

8. FAWCETT.D.W.: The structure of the mammalian spermatozoon. Internat. Rev. Cytol. 7, 195-234 (1958)

9. FORD. W.C.L \& A. HARRISON: The role of oxidative phosphorylation in the generation of ATP in human spermatozoa. J. Reprod. Fertil. 63, 271278 (1981)

10. FRASER, L.R. \& P.J. QuinN: A glycolytic product is obligatory for initiation of the sperm acrosome reaction and whiplash motility required for fertilization in the mouse. J. Reprod. Fertil. 61, 25-35 (1981)

II. HAMmerstedt, R.H. \& R.E. LoVRIEN: Calorimetric technique for metabolic studies of cells and 
organisms under normal conditions and stress. J. Exp. Zool. 228, $459-469$ (1983)

12. Hamner, R.S.. H. Goldman \& J.A. Mitchell: Effects of nicotine on intrauterine oxygen tension in the rat. J. Reprod. Fertil. 63, 163-168 (1981)

13. HARRISON, R.A.P., H.M. DOTT \& G.L. FOSTER: Bovine serum albumin, sperm motility, and the "dilution effect". J. Exp. Zool. 222, 81-88 (1982)

14. HefFNER, L.J.\& B.T.STOREY: The role of calcium in maintaining motility in mouse spermatozoa. J. Exp. Zool., 218, $427-434$ (1981)

15. HoPPE, P.C.: Glucose requirement for mouse sperm capacitation. Biol. Reprod., 15, 39-45 (1976)

16. Lardy, H.A. \& P.H. Phillips: The interrelationship of oxidative and glycolytic processes as sources of energy for bull spermatozoa. Amer. J. Physiol. 133, 602-609 (1941)

17. LindemanN, C.B. \& I.R. Gibbons: Adenosine triphosphate-induced motility and sliding of filaments in mammalian sperm extracted with Triton X-100. J. Cell Biol. 65, 147-162 (1975)

18. Linderstrom-LanG, K.: Principle of the Cartesian diver applied to gasometric technique. Nature 140 , p. 108 (1937)

19. ManN, T.P.: The Biochemistry of Semen and of the Male Reproductive Tract. Methuen and Co., London (1964)
20. Morton, B.E., C.F. Fraser \& R. Sagadraca: Initiation of hamster sperm motility from quiescence: effect of conditions upon flagellation and respiration. Fertil. Steril. 32, 222-227 (1979)

21. Peterson. R.N.\& M. Freund: ATP synthesis and oxidative metabolism in human spermatozoa. Biol. Reprod. 3, 47-54 (1970)

22. ROGERS, B.J., M. UENO\& R. YANAGIMACHI: Inhibition of hamster sperm acrosome reaction and fertilization by oligomycin, antimycin- $A$, and rotenone. J. Exp. Zool. 199, $129-136$ (1977)

23. RoGERS, B.J. \& R. YANIGAMACHI: Retardation of guinea pig acrosome reaction by glucose: the possible importance of pyruvate and lactate metabolism in capacitation and the acrosome reaction. Biol. Reprod. 13, 568-575 (1975)

24. Suter, P.Y., W. Chow \& I.C.A. Martin: Maintenance of motility in human spermatozoa by energy derived through oxidative phosphorylation. Biol. Reprod. 20, 505-510 (1979)

25. TAMPHAICHITR, N.: In vitro stimulation of human sperm motility by acetylcarnitine. Int. J. Fertil. 22, 85-91 (1977)

26. YoCHIM. J.M.\& J.A. MitCHELL: Intrauterine oxygen tension in the rat during progestation: its possible relation to carbohydrate metabolism and the regulation of nidation. Endocrinol. 83, 706713 (1968) 